\section{Fersensporn: wenn Injektionstherapie, dann am ehesten mit Botulinumtoxin}

\begin{abstract}
Eigentlich gilt die Plantarfasziitis („Fersensporn“) als selbstlimitierend: In 80$90 \%$ der Fälle gehen die Beschwerden therapieunabhängig innerhalb eines Jahres zurück. Fakt ist jedoch, dass die Patienten aufgrund der erheblichen Schmerzen sehr oft auf eine Behandlung drängen.
\end{abstract}

$E_{\text {in }}^{\text {in }}$ ne Injektionstherapie sollte bei einer Plantarfasziitis erst zum Einsatz kommen, wenn konservative Maßnahmen wie Kühlen, Schonen, Schuheinlagen und Physiotherapie nicht die ersehnte Linderung erzielen. Derzeit werden diverse Substanzen für die Injektionstherapie verwendet, darunter Kortikosteroide, NSAR, Botulinumtoxin A und plättchenreiches Plasma (PRP). Zur Bewertung des Behandlungserfolgs dieser Maßnahmen mangelt es jedoch vielfach an Studien mit hoher Qualität.

Mediziner aus Griechenland und der Schweiz haben nun in einer systematischen Literaturübersicht mithilfe einer Netzwerk-Metaanalyse die Ergebnisse von 22 randomisierten kontrollierten Studien (RCTs) ausgewertet. Die Analyse umfasste die Daten von 1.216 Patienten, die wegen ihrer Plantarfasziitis eine

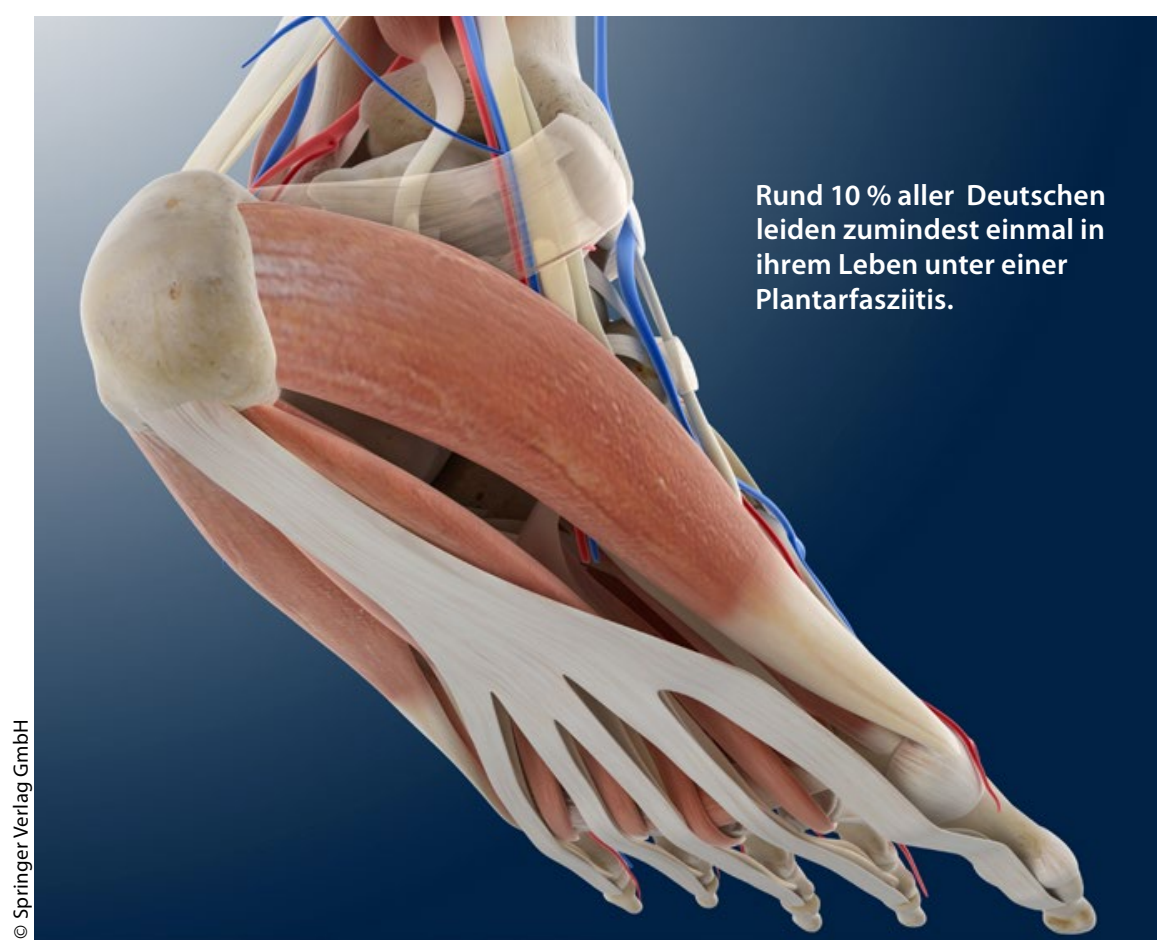

von elf verschiedenen Injektionstherapien erhalten hatten.

$\mathrm{Zu}$ Beurteilung der kurzfristigen Schmerzlinderung (bis zu zwei Monate nach Therapiebeginn) wurden 18 RCTs mit 1.009 Patienten herangezogen. Die meisten Daten (15\% der Gesamtmenge) waren zur Kortikosteroidtherapie verfügbar. Wie die Autoren berichten, waren Injektionen mit dehydrierter Amnionmembran, PRP oder Botulinumtoxin der Applikation von Kortikosteroiden jeweils signifikant überlegen; die mittleren Unterschiede auf der visuellen Analogskala betrugen $-7,32,-2,38$ beziehungsweise $-1,68$.

Eine kurzfristige Besserung der Funktionalität wurde vor allem mit Botulinumtoxin erzielt (vier Studien mit insfür die Grundlage). gesamt 180 Teilnehmern lieferten hier-

Wie es nach zwei bis sechs Monaten mit den Schmerzen aussah, leiteten die Forscher aus 15 Studien mit insgesamt 800 Teilnehmern ab. Hier lag Botulinumtoxin deutlich vorn. Nur in zwei RCTs waren die Patienten länger als sechs Monate (bis zu zwei Jahre) nachbeobachtet worden. In puncto Schmerzen zeichnete sich nach einem Jahr kein nennenswerter Unterschied zwischen NSAR und Kortikosteroid-Injektionen ab. PRP und eine Sklerosierungstherapie mit Dextrose lieferten nach 28 Wochen in etwa gleichwertige Ergebnisse. Schwere Nebenwirkungen wurden mit keiner der verglichenen Therapien berichtet.

Nach Tsikopoulos und Kollegen sind die positiven Ergebnisse zur dehydrierten Amnionmembran zwar vielversprechend; aufgrund der unzureichenden Datenlage vor allem bezüglich des mittelund längerfristigen Erfolgs halten sich die Autoren jedoch mit einer Empfehlung zurück. Anders bei Botulinumtoxin A: Mit dieser Substanz, so die Forscher, hätten die behandelten Patienten die vergleichsweise besten Chancen auf eine Besserung ihrer Schmerzen in einem Zeitfenster bis zu einem halben Jahr. Die Ergebnisse seien auch nach Herausrechnen von Verzerrungsrisiken robust geblieben.

Fazit: Wenngleich die Methode der Netzwerkanalyse ihre Mängel hat, ist doch das Konzept der BotulinumtoxinTherapie im Zusammenhang mit der Plantarfasziitis plausibel: So rührt der Fersenschmerz primär von einer Enthesiopathie am Ansatz der Plantarsehne am Calcaneus her. An der Pathologie beteiligt ist jedoch möglicherweise auch eine Tendinopathie des unter der Plantarfaszie gelegenen Musculus flexor digitorum brevis. Botulinumtoxin führt, an der richtigen Stelle injiziert, zur Relaxierung dieses Muskels. Damit bewirkt man, dass die Entzündung abklingen kann. Größere, von Herstellern unabhängige Studien müssen nun zeigen, ob das Verfahren generell für die Therapie des Fersensporns zu empfehlen ist.

Dr. Elke Oberhofer

Tsikopoulos K et al. Injection therapies for plantar fasciopathy ('plantar fasciitis'): a systematic review and network metaanalysis of 22 randomised controlled trials. Br J Sports Med 2016; online 3. Mai; doi: 10.1136/bjsports-2015-095437 